Pacific

Journal of

Mathematics

A WEAK MULTIPLICITY-ONE THEOREM FOR SIEGEL MODULAR FORMS

Rudolf Scharlau and Lynne H. Walling

Volume $211 \quad$ No. 2

October 2003 


\title{
A WEAK MULTIPLICITY-ONE THEOREM FOR SIEGEL MODULAR FORMS
}

\author{
Rudolf Scharlau and Lynne H. Walling
}

\begin{abstract}
Using the explicit action of the Hecke operators $T(p)$ acting on the Fourier coefficients of Siegel modular forms of arbitrary degree and level, a short and elementary proof and a generalization of a result by Breulmann and Kohnen is obtained, which says that cuspidal eigenforms are determined by their coefficients on matrices of square-free content.
\end{abstract}

\section{Introduction.}

In a recent paper by Breulmann and Kohnen [BK99], the authors obtain a weak multiplicity-one result on (integral weight) Siegel-Hecke cuspidal eigenforms of degree 2, showing that such forms are completely determined by their coefficients on matrices of the form $m S$, where $S$ is primitive and $m$ is square-free. To show this, they twist Andrianov's identity relating the Maaß-Koecher series and the spinor zeta function of an eigenform [An74] by a Größencharacter. This allows them to then use Imai's converse theorem for degree 2 forms [Im80] and thereby obtain their result.

In this note, we use an elementary algebraic argument to reprove and extend their result to Siegel modular forms of arbitrary degree $n$ and arbitrary level which are only assumed to be eigenforms for the operators $T(p)$ (but not necessarily for the full Hecke algebra). We first show that such an eigenform must have primitive matrices in the support of its Fourier development. Then it is immediate from the explicit action of the Hecke operators on Fourier coefficients that if two such forms have the same eigenvalues for all $T(p)$ and the same coefficients on primitive matrices then their difference must be zero. Since, moreover, the assumption of coinciding eigenvalues can be derived from the above stated assumption of Breulmann and Kohnen, we recover their result for $n=2$.

Note that Andrianov's identity and Imai's converse theorem are currently only known for $n=2$ and level 1, so the analytic approach used in [BK99] cannot at this time be extended to general $n$. 


\section{Preliminaries.}

Let $F$ be a degree $n$ Siegel modular form with Fourier expansion

$$
F(\tau)=\sum_{S} c(S) e\{S \tau\}
$$

where $S$ runs over all symmetric positive semidefinite even integral $n \times n$ matrices $S$ and $e\{\tau\}=\exp (\pi i$ trace $\tau)$. We consider each $S$ to be a quadratic form on a $\mathbb{Z}$-lattice $\Lambda$ of rank $n$ relative to some basis for $\Lambda$. As $S$ varies, the pair $(\Lambda, S)$ varies over all isometry classes of rank $n$ lattices with even integral positive semi-definite quadratic forms. Also, the isometry class of $(\Lambda, S)$ is that of $\left(\Lambda, S^{\prime}\right)$ if and only if $S^{\prime}=S[G]=G^{t} S G$ for some $G \in \mathrm{GL}_{n}(\mathbb{Z})$. When $k$ is even, $F(\tau[G])=F(\tau)$ for all $G \in \mathrm{GL}_{n}(\mathbb{Z})$, so it follows that $c(S[G])=c(S)$. Hence, (with $k$ even) we can rewrite the Fourier expansion of $F$ in the form

$$
F(\tau)=\sum_{\operatorname{class} \Lambda} c(\Lambda) e^{*}\{\Lambda \tau\}
$$

where $c(\Lambda)=c(S)$ for any matrix $S$ representing the quadratic form on $\Lambda$, and with $\mathrm{O}(\Lambda)$ the orthogonal group of $\Lambda$ we set

$$
e^{*}\{\Lambda \tau\}=\sum_{G \in \mathrm{O}(\Lambda) \backslash \mathrm{GL}_{n}(\mathbb{Z})} e\{S[G] \tau\} .
$$

When $k$ is odd, we have $F(\tau[G])=\operatorname{det} G \cdot F(\tau)$, so $c(S[G])=\operatorname{det} G \cdot c(S)$, and a completely analogous formula holds with $\Lambda$ considered as an oriented lattice (i.e., a pair consisting of a lattice and one of the two orientation classes of its bases), and the sum in the definition of $e^{*}$ being over $\mathrm{SO}(\Lambda) \backslash \mathrm{SL}_{n}(\mathbb{Z})$.

In what follows we make use of the 'content' and the 'discriminant' of lattice. When $\Lambda$ is a lattice with quadratic form $q$, the content cont $\Lambda$ of $\Lambda$ is defined as

$$
\operatorname{cont} \Lambda:=\operatorname{gcd}\{q(x, x) / 2 \mid x \in \Lambda\} .
$$

If $q$ on $\Lambda$ has the Gram matrix $S$, with respect to some basis, then cont $\Lambda$ is just the gcd of the entries $s_{i j}, i \neq j, s_{i i} / 2$ of $S$. (The term 'content' is standard for symmetric matrices, but not for lattices; 2 cont $\Lambda$ is equal to what is usually called the 'norm' of the lattice $\Lambda$; see [O'M71] for further information.) The determinant of $S$ does not depend on the choice of the basis and is called the discriminant $\operatorname{disc} \Lambda$ of $\Lambda$. It is zero if the lattice (i.e., its quadratic form) is degenerate. In this case, $q$ induces a nondegenerate form on $\bar{\Lambda}:=\Lambda / \operatorname{rad} \Lambda$, where $\operatorname{rad} \Lambda \subseteq \Lambda$ denotes the radical of $q$. The dimension of $\bar{\Lambda}$ is also called the rank of $\Lambda$, or rather of $(\Lambda, q)$. We write

$$
\operatorname{rank} \Lambda:=\operatorname{dim} \bar{\Lambda}=\operatorname{dim} \Lambda-\operatorname{dim} \operatorname{rad} \Lambda .
$$

For a positive rational number $\alpha$, the notation $\Lambda^{\alpha}$ means we "scale" $\Lambda$, or rather the pair $(\Lambda, q)$, by $\alpha$, i.e., $\Lambda^{\alpha}$ is equipped with the quadratic form $\alpha q$. 
We summarize here the results on content, scaling and discriminant used in the proofs below; $\Lambda$ is a lattice of rank $r$ and $\Omega$ a sublattice of finite index.

- $[\Lambda: \Omega]=m \Longrightarrow \operatorname{disc} \Omega=m^{2} \operatorname{disc} \Lambda$.

- $\operatorname{disc} \Lambda^{\alpha}=\alpha^{r} \operatorname{disc} \Lambda$.

- $\operatorname{cont} \Lambda^{\alpha}=\alpha \operatorname{cont} \Lambda$.

The first formula is well-known and is verified e.g., by taking a pair of elementary divisor bases of $\Omega \subseteq \Lambda$ and their corresponding Gram matrices; the other two formulas are obvious.

We now recall from e.g., [Fr83], Kapitel IV, the notion of the Hecke operators $T(p)$, for all primes $p$, acting on Siegel modular forms degree $n$, level $\ell$ and character $\chi$ (and any fixed weight $k$ ). The Siegel modular form $T(p) F$ or $F \mid T(p)$, with $F$ as above, is defined by averaging $F$ over the double coset $\Gamma_{n}(\ell) g \Gamma_{n}(\ell)$ of the rational symplectic similitude $g=\left(\begin{array}{cc}p I_{n} & 0 \\ 0 & I_{n}\end{array}\right)$, where

$$
\Gamma_{n}(\ell)=\left\{\left(\begin{array}{ll}
A & B \\
C & D
\end{array}\right) \in \operatorname{Sp}_{n}(\mathbb{Z}) \mid C \equiv 0 \quad(\bmod \ell)\right\} .
$$

See e.g., $[\mathbf{F r} \mathbf{8 3}]$ for the precise definition (and for the definition of the other Hecke operators $T_{j}\left(p^{2}\right), j=1, \ldots, n-1$, which apparently cannot be used to improve the result below).

We denote by $T(p) c(\Lambda)$ the $\Lambda$ 'th Fourier coefficient of $T(p) F$. In $\S 4$ and $\S 6$ of $[\mathbf{H a W a}]$, it is shown that, for $p$ prime,

$$
T(p) c(\Lambda)=\sum_{p \Lambda \subsetneq \Omega \subseteq \Lambda} \gamma(\Omega) c\left(\Omega^{1 / p}\right)+c\left(\Lambda^{p}\right),
$$

with

$$
\gamma(\Omega)=\chi\left(p^{n-j}\right) p^{k(n-j)+j(j+1) / 2-n(n+1) / 2} \text { where }[\Lambda: \Omega]=p^{j} .
$$

For level $\ell=1$, this description of $T(p)$ is essentially already contained in the classical work [Ma51] by Maaß; it is also readily derived from the wellknown coset representatives for the above double coset, as described e.g., in [Fr83], Kapitel 4. The generalization to arbitrary $\ell$ is easy since we are only dealing with the $T(p)$; notice that the sum over $\Omega$ in the above formula disappears for $p \mid \ell$.

When $F$ is an eigenform and $T(p) F=\lambda_{F}(p) F$, we shall refer to this formula as the 'Hecke eigenform equation'.

\section{The result.}

We immediately proceed to our main result.

Theorem 3.1. Suppose $F, G$ are degree $n$ eigenforms of arbitrary level and character, for all $T(p)$, with the same eigenvalues (i.e., $\lambda_{F}(p)=\lambda_{G}(p)$ for all $p)$, and that their Fourier coefficients agree on primitive lattices and on 0 . Then $F=G$. 
Proof. By the support supp $F$ of a Siegel modular form as above, we mean the support of its Fourier coefficients, i.e., the set of lattices $\Lambda$ with $c(\Lambda) \neq 0$. Suppose $F \neq G$. Then $F-G$ is an eigenform for all $T(p)$ with no primitive lattice in its support. But this is impossible by the following lemma:

Lemma 3.2. Let $F$ be a degree $n$ eigenform for $T(p)$ for all primes $p$. Then there is at least one primitive lattice in the support of $F$.

Proof. Suppose the contrary. For $1 \leq r \leq n$ let

$$
\operatorname{supp}_{r} F=\{\Lambda \in \operatorname{supp} F \mid \operatorname{rank} \Lambda=n-\operatorname{dim} \operatorname{rad} \Lambda=r\} .
$$

We first consider the case $\operatorname{supp}_{n} F \neq \emptyset$. Let $N$ be the minimal content of lattices in $\operatorname{supp}_{n} F$ (so $N>1$ ). Take a prime $p \mid N$. Then among the lattices in $\operatorname{supp}_{n} F$ with content $N$, choose $\Lambda$ s.t. the $p$-part of $\operatorname{disc} \Lambda$ is minimal. Since $\Lambda^{1 / p}$ is integral, the Hecke eigenform equation says

$$
\lambda_{F}(p) c_{F}\left(\Lambda^{1 / p}\right)=\sum_{p \Lambda \subsetneq \Omega \subseteq \Lambda} \gamma(\Omega) c_{F}\left(\frac{1}{p} \Omega\right)+c_{F}(\Lambda) .
$$

For $\Omega$ s.t. $p \Lambda \subsetneq \Omega \subseteq \Lambda$, we have $\Lambda \subsetneq \frac{1}{p} \Omega$ and hence the $p$-part of $\operatorname{disc}\left(\frac{1}{p} \Omega\right)$ is strictly smaller than that of $\operatorname{disc} \Lambda$. Similarly, $\operatorname{disc} \Lambda^{1 / p}=p^{-n} \operatorname{disc} \Lambda$. Hence $\Lambda^{1 / p}, \frac{1}{p} \Omega \notin \operatorname{supp}_{n} F$, for $p \Lambda \subsetneq \Omega \subseteq \Lambda$; so the Hecke eigenform equation says $0=c_{F}(\Lambda)$, contradicting that $\Lambda$ was chosen in $\operatorname{supp}_{n} F$.

Now we consider the case $\operatorname{supp}_{n} F=\emptyset$ and let $r$ be maximal with $\operatorname{supp}_{r} F \neq \emptyset$. Note that $r>0$. We use Siegel's $\Phi$-operator (see [Fr83], Kapitel IV , §4) to reduce this case to the previous one. Indeed, this operator lowers the degree by 1 and commmutes up to a constant with $T(p)$, thus $G:=F \mid \Phi^{n-r}$ is a degree $r$ eigenform for all $T(p)$. Moreover

$$
\operatorname{supp}_{r} F=\left\{\Lambda \perp \mathbf{0}_{n-r} \mid \Lambda \in \operatorname{supp}_{r} G\right\} .
$$

Since, by the preceding case, $\operatorname{supp}_{r} G$ contains a primitive lattice, so does $\operatorname{supp}_{r} F$.

The next lemma shows, for cusp forms, the equivalence between our assumption of coinciding eigenvalues and the assumption used in [BK99].

Lemma 3.3. Let $F, G$ be degree $n$ cuspidal eigenforms for each $T(p), p$ prime, s.t. the coefficients of $F$ and $G$ agree on primitive lattices. Then $\lambda_{F}(p)=\lambda_{G}(p)$ for all $p$ if and only if the coefficients of $F, G$ agree on all primitive lattices scaled by non-squares.

Proof. Suppose $\lambda_{F}(p)=\lambda_{G}(p)$ for all $p$. Let $Q \in \mathbb{N}$ be square-free, and let $p$ be a prime not dividing $Q$. Suppose we know that the coefficients of $F, G$ agree on all primitive lattices scaled by divisors of $Q$. (Note that we are assuming this for $Q=1$.) We show that the coefficients of $F, G$ must then agree on all primitive lattices scaled by divisors of $p Q$. 
Let $\Lambda$ be a primitive lattice scaled by some divisor of $Q$ s.t. $p \nmid \operatorname{disc} \Lambda$. (Note that disc $\Lambda \neq 0$ since $F$ is cuspidal.) Then for $p \Lambda \subsetneq \Omega \subseteq \Lambda$, we have $[\Lambda: \Omega]=p^{s}$ with $s<n$. Hence $\operatorname{disc} \Omega=p^{2 s} \cdot \operatorname{disc} \Lambda$, so $p^{2 s} \| \operatorname{disc} \Omega$. Thus $p^{2} \nmid \operatorname{cont} \Omega$ (else $p^{2 n} \mid \operatorname{disc} \Omega$ ), so $\Omega$ is a primitive lattice scaled by some divisor of $p Q$. This means either $\Omega^{1 / p}$ is not integral or is a primitive lattice scaled by a divisor of $Q$; in either case $c_{F}\left(\Omega^{1 / p}\right)=c_{G}\left(\Omega^{1 / p}\right)$. This together with the Hecke eigenform equation then gives us

$$
\begin{aligned}
c_{F}\left(\Lambda^{p}\right) & =\lambda_{F}(p) c_{F}(\Lambda)-\sum_{p \Lambda \subsetneq \Omega \subseteq \Lambda} \gamma(\Omega) c_{F}\left(\Omega^{1 / p}\right) \\
& =\lambda_{G}(p) c_{G}(\Lambda)-\sum_{p \Lambda \subsetneq \Omega \subseteq \Lambda} \gamma(\Omega) c_{G}\left(\Omega^{1 / p}\right) \\
& =c_{G}\left(\Lambda^{p}\right) .
\end{aligned}
$$

Now suppose that for some $t \geq 1$ we know the coefficients of $F, G$ agree on primitive lattices $\Delta$ scaled by a divisor of $p Q$ provided $p^{t} \nmid \operatorname{disc} \Delta$. Let $\Lambda$ be a primitive lattice scaled by a divisor of $Q$ s.t. $p^{t} \|$ disc $\Lambda$. Take $\Omega$ s.t. $p \Lambda \subsetneq$ $\Omega \subseteq \Lambda$. Since $p^{2} \| \operatorname{cont}(p \Lambda)$ and cont $\Omega \mid \operatorname{cont}(p \Lambda)$, we have $p^{3} \nmid \operatorname{cont} \Omega$. Thus $\frac{1}{p} \Omega$ is either non-integral, or primitive scaled by some divisor of $p Q$. Also since $p \Lambda \subsetneq \Omega$, we know $[\Lambda: \Omega]=p^{r}$ for some $r<n$, so $p^{2(r-n)+t} \| \operatorname{disc} \frac{1}{p} \Omega$. Hence by hypothesis, $c_{F}\left(\frac{1}{p} \Omega\right)=c_{G}\left(\frac{1}{p} \Omega\right)$. Consequently, the Hecke eigenform equation, with all terms rescaled by $\frac{1}{p}$, gives us $c_{F}(\Lambda)=c_{G}(\Lambda)$.

Induction on $t$ shows that $c_{F}(\Lambda)=c_{G}(\Lambda)$ for all $\Lambda$ that are primitive lattices scaled by a divisor of $p Q$. Induction on the number of primes dividing $Q$ shows $c_{F}(\Lambda)=c_{G}(\Lambda)$ for all $\Lambda$ that are primitive lattices scaled by nonsquares.

Conversely, suppose the coefficients of $F, G$ agree on all primitive lattices scaled by non-squares. Fix a prime $p$. Choose a primitive lattice $\Lambda \in \operatorname{supp} F$. Then as shown above, for $\Omega$ s.t. $p \Lambda \subsetneq \Omega \subseteq \Lambda, \Omega^{1 / p}$ is either non-integral or a primitive lattice scaled by a non-square. Thus

$$
\sum_{\Omega} \gamma(\Omega) c_{F}\left(\Omega^{1 / p}\right)+c_{F}\left(\Lambda^{p}\right)=\sum_{\Omega} \gamma(\Omega) c_{G}\left(\Omega^{1 / p}\right)+c_{G}\left(\Lambda^{p}\right),
$$

so the Hecke eigenform equation implies

$$
\lambda_{F}(p) c_{F}(\Lambda)=\lambda_{G}(p) c_{G}(\Lambda) .
$$

Also $c_{F}(\Lambda)=c_{G}(\Lambda)$ by hypothesis, and $c_{F}(\Lambda) \neq 0$. Hence $\lambda_{F}(p)=\lambda_{G}(p)$.

Remark 3.4. Using the $\Phi$-operator as before, one can easily remove the restriction to cusp forms in the previous lemma. 


\section{References}

[An74] A.N. Andrianov, Euler products corresponding to Siegel modular forms of genus 2, Russ. Math. Surveys, 29:3 (1974), 43-110, MR 55 \#5540, Zbl 0304.10021.

[BK99] S. Breulmann and W. Kohnen, Twisted Maaß-Koecher series and spinor zeta functions, Nagoya Math. J., 155 (1999), 153-160, MR 2000g:11033, Zbl 0936.11030.

[Fr83] E. Freitag, Siegelsche Modulfunktionen, Springer-Verlag Berlin, 1983, MR 88b:11027, Zbl 0498.10016.

[HaWa] J.L. Hafner and L.H. Walling, Explicit action of Hecke operators on Siegel modular forms, J. Number Theory, 93 (2002), 34-57, MR 2003a:11052.

[Im80] K. Imai, Generalization of Hecke's correspondence to Siegel modular forms of degree 2, Amer. J. Math., 102 (1980), 903-936., MR 82e:10045, Zbl 0447.10028.

[Ma51] H. Maaß, Die Primzahlen in der Theorie der Siegelschen Modulfunktionen, Math. Ann., 124 (1951), 87-122, MR 13,823g, Zbl 0044.30901.

[O'M71] O.T. O'Meara, Introduction to Quadratic Forms, Springer-Verlag Berlin, 1971, MR 50 \#269, Zbl 0207.05304.

Received July 7, 2000.

FACHBEREICH MATHEMATIK

UNIVERSität DORTMUND

44221 DorTMund

Germany

E-mail address: scharlau@math.uni-dortmund.de

Department of Mathematics

UNIVERSITY OF COLORADO

Boulder, CO 80309

E-mail address: walling@euclid.colorado.edu 